\title{
A Path Generation Method for Wire and Arc Additive Remanufacturing of Complex Hot-forging Dies
}

\section{Yonghua Shen}

College of Material Science and Technology, Nanjing University of Aeronautics and Astronautics, Nanjing 211106, China

\section{Yanhong Wei}

College of Material Science and Technology, Nanjing University of Aeronautics and Astronautics, Nanjing 211106, China

Renpei Liu ( $\square$ nuaaliurenpei@126.com )

Nanjing University of Aeronautics and Astronautics

\section{Research Article}

Keywords: Additive remanufacturing, Path generation, Forging die, Depositing quality

Posted Date: March 15th, 2021

DOl: https://doi.org/10.21203/rs.3.rs-285538/v1

License: (c) (1) This work is licensed under a Creative Commons Attribution 4.0 International License. Read Full License

Version of Record: A version of this preprint was published at The International Journal of Advanced Manufacturing Technology on August 13th, 2021. See the published version at https://doi.org/10.1007/s00170-021-07813-w. 


\title{
A path generation method for wire and arc additive remanufacturing of complex hot-forging \\ dies
}

\author{
Yonghua Shen, Yanhong Wei, Renpei Liu \\ College of Material Science and Technology, Nanjing University of Aeronautics and Astronautics, \\ Nanjing 211106, China \\ E-mail: Yonghua Shen 15295750982@163.com \\ Yanhong Wei nuaadw@126.com \\ Renpei Liu_nuaaliurenpei@126.com
}

\begin{abstract}
Wire and arc additive remanufacturing (WAAR) technology has become a new solution for hot-forging dies repair and remanufacturing. In this study, a path generation method is proposed for WAAR of hot-forging dies. At first, a WAAR process of the hot-forging die is presented, and considering the characteristics of large welding heat input and complex 3D digital model, the hybrid path planning strategy is confirmed as an appropriate strategy for WAAR. The developed hybrid path generation method for WAAR consists of three main steps: determinate the direction of the scan line; divide and fill the internal area; and connect the sub-paths. the relatively optimal scanning direction is determined by calculating the length and inclination angle of each line segment in the contour lines, which reduces the possibility of sharp angles. The internal region is divided based on the location of the selected extreme points, and the path space is adjusted to avoid the occurrence of the underfilled phenomenon. At the stage of sub-paths connection, some criteria are proposed to reduce the number of sub-paths. At last, a planar deposition experiment and the WAAR process of four damaged hotforging dies are carried out to validate the effectiveness and robustness of the proposed method.
\end{abstract}

Keywords Additive remanufacturing; Path generation; Forging die; Depositing quality

\section{Introduction}

In the past few decades, researchers have shown an increased interest in additive manufacturing (AM) technology due to its distinguished advantage of design flexibility, material saving, and time efficiency[1][2][3]. Gradually, AM technology has been increasingly adopted in the aerospace, medical, and automotive industries[4][5]. As one of the AM processes, Wire and arc additive manufacturing (WAAM) fabricates metallic 
components by depositing beads of weld metal layer-by-layer, using existing arc welding process such as the gas tungsten arc welding (GTAW), the gas metal arc welding (GMAW), etc. [6][7][8]. Compared with other AM processes, WAAM is now widely accepted as a more appropriate method for manufacturing large-scale components due to its higher deposition rate and lower costs[9][10]. In addition, WAAM technology has recently been found to be suitable for remanufacturing largescale industrial hardware such as hot-forging dies, which is urgent for market demand.

In general, a WAAM process consists of four essential steps, namely, the building of a 3D model, slicing, path generation, and process parameters designing[11]. The contours of each layer can be obtained from slicing the 3D model, and a tool-path is the trajectory of the welding torch to fill the interior areas of each layer according to these contours. As the path generation in the process plays a crucial role in the building efficiency and geometrical quality, developing appropriate path planning algorithms to satisfy different requirements has always been a research hotspot. Direction-parallel paths and contour-parallel paths are the most commonly employed path patterns in WAAM technology[12]. The generation algorithm of direction-parallel paths is easy and fast to realize, but it always leads to an unsatisfactory fabrication accuracy due to discretization errors. In contrast, the contour-parallel paths have the advantages of high fabrication accuracy, while the offset algorithm is rather complicated especially when the shapes of the boundaries are comparatively complex. Furthermore, gaps may appear during the depositing process in this pattern. Several path strategies have been proposed based on these two patterns. Zhang et al. put forward a hybrid path planning strategy for WAAM[13]. The outline vector path is used to fill the outline of the image to obtain satisfied surface accuracy, while the interior is filled with the zigzag path which is faster and universal. Ding et al. proposed a MAT (Medial Axis Transformation) path planning methodology for the WAAM process[12]. This path strategy can tackle the gap problem completely by offsetting the medial axis of the cross-sectional geometry towards its boundary. Nevertheless, the MAT-based path was proved to be more suitable for WAAM of thin-walled structures and the outline accuracy of solid structures is still poor. Besides, to fulfil the requirements of tool-paths for WAAM including continuities, high geometrical accuracy, and simple implementation, a novel path generation strategy has been introduced by Ding et al[14]. The strategy is able to generate one closed curve that can fill the given 2D geometry. Similarly, early studies by Dwivedi et al. have developed a continuous path planning strategy for WAAM[15]. Both of them have the same limitation, that is, they cannot be effective and robust on arbitrarily shaped geometries. As opposed to the approach that generates paths using the same path strategy, an alternative approach is designing different path strategies according to the diverse geometric feature of parts, such as cross structures[6], T-crossing features[16] and so on. Furthermore, Michel et al. introduced a new path planning solution called Modular Path Planning (MPP)[17]. The MPP solution combining the efficiency of the layer-by-layer deposition strategy to the adaptability of the feature-based approach can guarantee a more uniform deposition in WAAM. However, this kind of approach still requires excessive user intervention and is particularly beneficial for WAAM of thinwalled structures compare with solid structures. 
Compared with the general WAAM process, the wire and arc additive remanufacturing (WAAR) of complex hot-forging die generally adopts larger heat input considering deposition efficiency, which makes the collapse of the deposited metal and unevenness phenomenon more prone to occur. Moreover, the digital model in the WAAR process of hot-forging dies is more complex and diverse. Hence, it is necessary to design corresponding path strategies for WAAR according to its specific characteristics. However, the above-described path strategies were mainly developed for WAAM and have their own advantages and limitations, little attention has been paid to WAAR. This paper proposes a path generation method for WAAR of complex hotforging dies to improve depositing quality. The remaining part of the paper proceeds as follows. Section 2 presents the remanufacturing processes of hot-forging dies and figures out the special requirements of path planning for WAAR. In Section 3, a hybrid path generation method is proposed in detail. The strategy consists of three main steps: determinate the direction of the scan line, divide and fill the internal area and connect the sub-paths. The implementation and the discussions of the proposed strategy are presented in Section 4, followed by a conclusion in Section 5.

\section{WAAR of hot-forging dies}

\section{The remanufacturing processes}

Hot-forging dies need to be in direct contact with high-temperature metals during the work process, and at the same time bear huge impact loads[18]. The working environment is relatively harsh, so it is prone to many forms of failure such as cracking, wear, deformation and fatigue[19]. To improve the service time of the hot-forging die, the GMAW-based manual surfacing welding method is usually used in the production practice to repair and remanufacture the hot-forging die[20]. It can produce new properties on the surface of the die which improves toughness, hardness and strength. However, the method has a poor working environment, and the accuracy and quality of the die after surfacing are directly related to the level of the welding worker, which will cause waste of materials and increase in cost.

The wire and arc additive remanufacturing technology has become a new solution for hot-forging dies repair and remanufacturing. With this technology, the repair and remanufacturing process can be automated, while saving a large number of welding materials, and improving production efficiency. As shown in Fig. 1, a wire and arc additive remanufacturing process of hot-forging dies is as follows:

1. Gouging: After surface cleaning of the damaged die (Fig. 1a), the defective region of the die is usually removed by arc gouging (Fig. 1b). The reason why the arc gouging method is chosen instead of the machine milling method is because of the consideration of costs and efficiency.

2. 3D scanning: Scan the gouged die with a $3 \mathrm{D}$ scanner to get the point cloud data (Fig. 1c).

3. 3D reconstruction: With the help of some reverse engineering software, like 
Imageware or Geomagic, the 3D model of the gouged die is reconstructed based on the point cloud data (Fig. 1d).

4. Boolean operations: Perform a Boolean operation on the gouged die model and the original intact die model to obtain the model (WAAR model) representing the removed part of the die (Fig. 1e).

5. Slicing and path generation: The WAAR model is usually sliced from the bottom to the top with the preset thickness, and each resulting layer adopts the same path generation strategy (Fig. 1f).

6. Simulation: Perform off-line simulation of robot motion in specific software to check the rationality of robot motion and avoid undesirable situations (Fig. $1 \mathrm{~g})$.

7. Metal deposition: Translate the path information into the program that can be executed by the specified robot. And then, the metal deposition process by GMAW is performed with preset welding parameters (Fig. 1h).

8. After the die is repaired, heat treatment and milling are required. The former aims to improve its structure and performance, and the latter is used to restore its size.

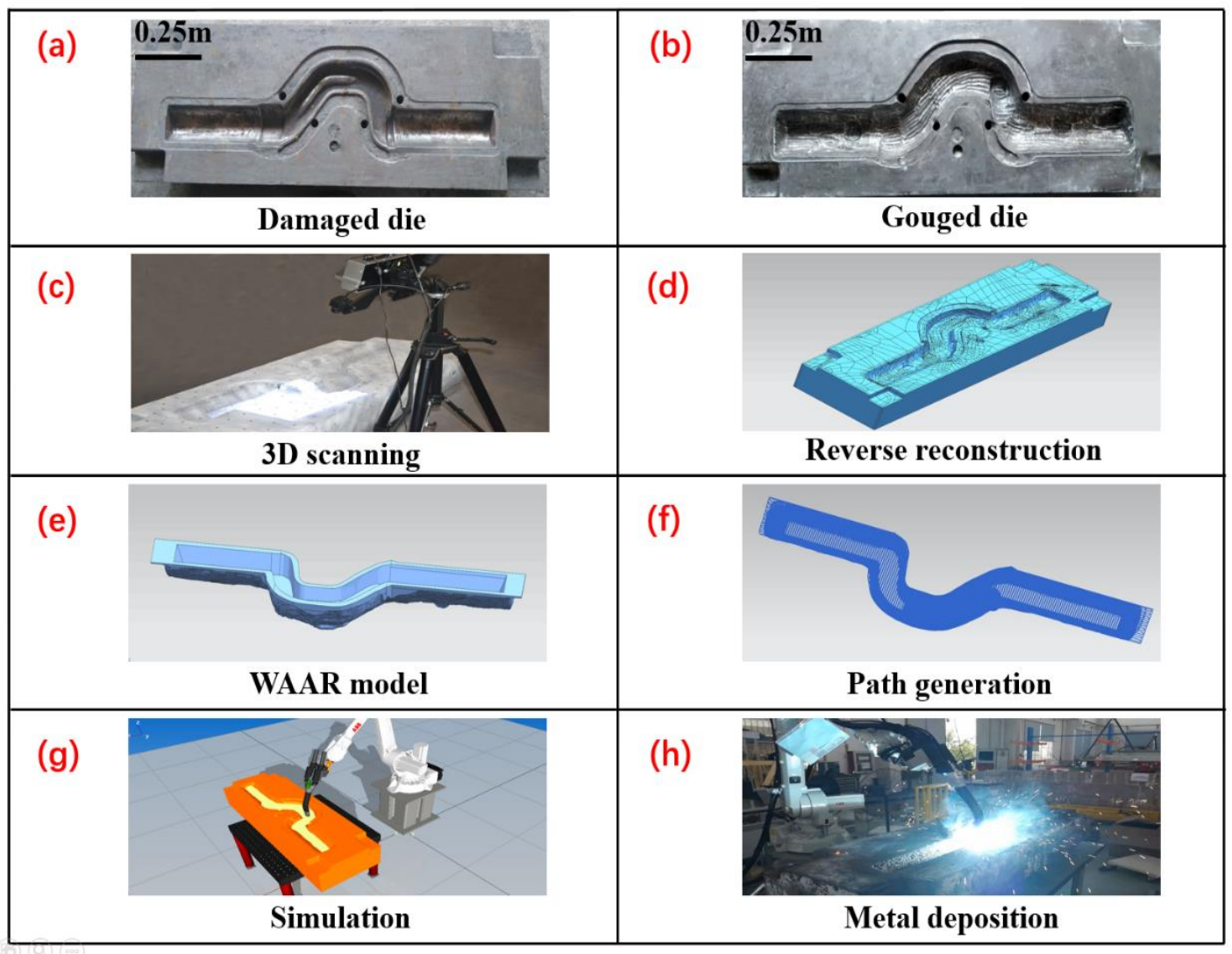

Fig. 1 The WAAR process of the hot-forging die

\section{Path planning strategy for WAAR}

The hybrid path planning strategy that generating contour paths along the boundary firstly and then filling the internal area with zigzag paths, is considered the 
most appropriate strategy for WAAR. The specific reasons for choosing this strategy are stated as follows: the first is that the contour paths (including inner and outer boundaries) can ensure that the deposited metal obtain satisfied dimensional accuracy; the second is that the paths generated by this strategy can be applied to the arbitrary geometric shape. Generally, the shape of the hot-forging dies cavity surface is complex. Moreover, due to the manual gouging process, this problem becomes worse. Consequently, the adopted algorithms must be effective and universal for arbitrary geometry; and the third is that filling the boundaries of contours first can avoid incomplete fusion and the weld bead collapse. As shown in Fig.2, the part of the contour paths close to the die base can ensure the fusion between the deposited metal and the die base. And if only the zigzag paths are used, it is likely to result in incomplete fusion defects. On the other hand, when filling the internal area, at the position away from the die base, due to the large welding heat input, the liquid metal in the molten pool cannot solidify rapidly and flows around, and finally, the weld bead may collapse. In this case, the contour paths can act as a barrier to prevent this phenomenon from happening.

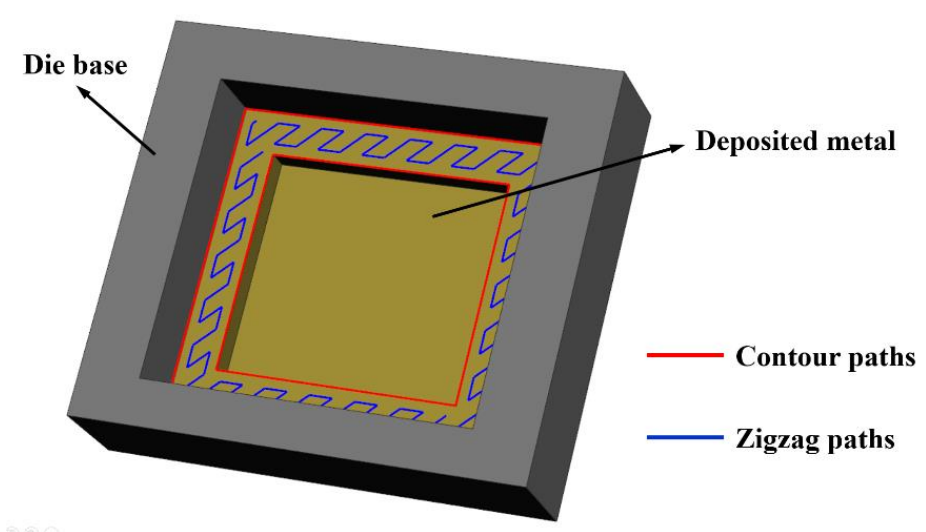

Fig. 2 The hybrid path planning strategy

When adopting the hybrid path planning strategy in a WAAR process, it is worth noting that overfilled or underfilled phenomenon is prone to occur in the area close to the contour line. One of the cases occurs at the location where two direction-parallel line segments are connected. As shown in Fig.3, The underfilled area is approximately regarded as a triangle region, and the overfilled area is the same according to geometric symmetry. The area of this triangle region is calculated as Eq. (1):

$$
S_{\text {overfilled }}=S_{\text {underfilled }}=\frac{d^{2} * \cot \alpha}{2}
$$

Where $d$ is the path space and $\alpha$ is the angle between the scan line and the contour line. It is clear that, the smaller the angle $\alpha$, the more serious the overfilled or underfilled phenomenon. Especially, when the angle $\alpha$ is $\pi / 2$ (the scan line is perpendicular to the contour line), it can be considered that there is no overfilled or underfilled phenomenon. 


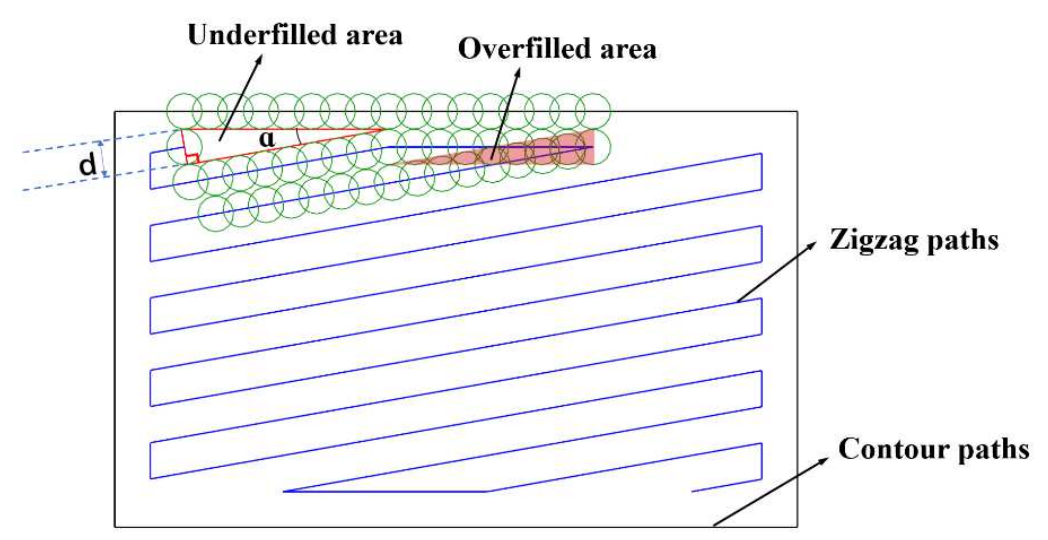

Fig. 3 The overfilled and underfilled phenomenon in corners

Besides, the path space of the WAAR process keeps constant generally, which may lead to another case of underfilled. Since the internal zigzag path and the contour path must be separated by a path space, the internal path is generated by the intersection of the scan lines and the offsetting contour lines instead of the contour lines. Hence, the distance between the extreme point of the contour line and its adjacent scan line is always greater than the path space, which means that there must be an underfilled area between them, as shown in Fig. 4.

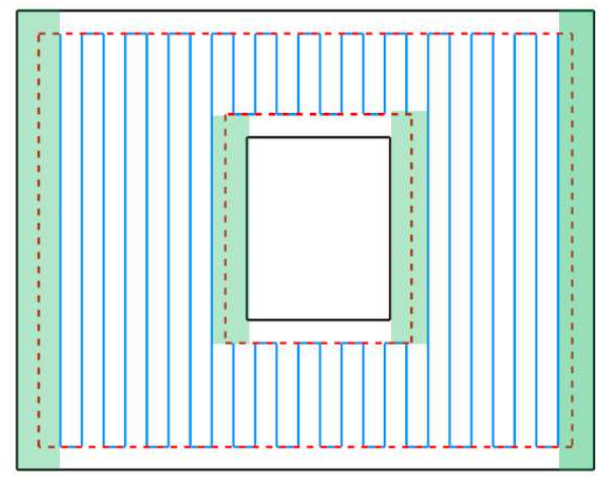

— Contour line(path)

Offsetting contour line

Internal zigzag path

Underfilled area

Fig. 4 Illustration of the underfilled phenomenon

It is generally believed that overfilled or underfilled phenomenon will make the deposition quality worse. Since the deposited metal cannot reach the corners of the underfilled area, voids may be produced during the process; and when the next layer of metal is deposited, weld bead collapse is likely to occur in the overfilled area. In particular, in the WAAR process of hot-forging dies, the diameter of the welding wire and the welding heat input are generally large, which leads to a relatively large width of the single-pass weld bead (typically greater than $10 \mathrm{~mm}$ ). This means that the overfilled or underfilled phenomenon in WAAR could be a severe problem. In addition, the weld bead geometry is usually uneven at the start and end of the weld paths. Moreover, in the WAAR process of hot-forging dies, to avoid collisions with the die base, the welding torch must be raised to a certain height after each welding pass is deposited. Hence, the number of path passes should be minimized to improve manufacturing efficiency and quality. The path generation method introduced in this 
paper focuses on dealing with the issues described above.

\section{Practical path generation method for WAAR}

\section{Determination of the direction of the scan line}

According to the requirements of path planning for WAAR, the contour path needs to be implemented first, and then the internal path follows. The contour lines of each layer are obtained after the model is sliced with the selected layer thickness. Obviously, the contour path consists of a number of straight-line segments. And as explained previously, the internal zigzag path is generated by the intersection of the scan lines and the offsetting contour lines. In this stage, the direction of the scan line has a great influence on the generated path. First, it affects the size of the underfilled and overfilled areas at the corners of the path. In addition, it also affects the number of sub-regions, which means the number of weld passes may increase. Compared with the latter, the former should be given priority because it may prevent the deposition process from continuing. However, due to the complex and irregular contour shapes, it is difficult to find an optimal direction to minimize the overfilled or underfilled area.

In fact, only sharp corners in the path may cause severe forming problems[21][22]. A sharp corner will appear usually when the angle between the scan line and the contour line is small. Hence, a compromise is to find the relatively optimal direction of the scan line so that the possibility of sharp corners is greatly reduced.

Take the contour lines shown in Fig. 5 as an example to introduce a method for selecting the direction of the scan line, and the inclination angle $\theta$ between the scan line and the positive direction of the $\mathrm{x}$-axis represents the direction of the scan line. The method is as follows: firstly, calculate the length of each line segment in the contour lines and the angle between it and the x-axis. The angle ranges from $0^{\circ}$ to $180^{\circ}$, which is divided into six intervals on average. Secondly, count the total length of line segments in each interval. Finally, select the interval with the minimum total length of the line segments, and the inclination angle $\theta$ is equal to the middle value of this interval. In particular, when the selected interval has only one line segment, the inclination angle $\theta$ is equal to the angle between the line segment and the $\mathrm{x}$-axis.

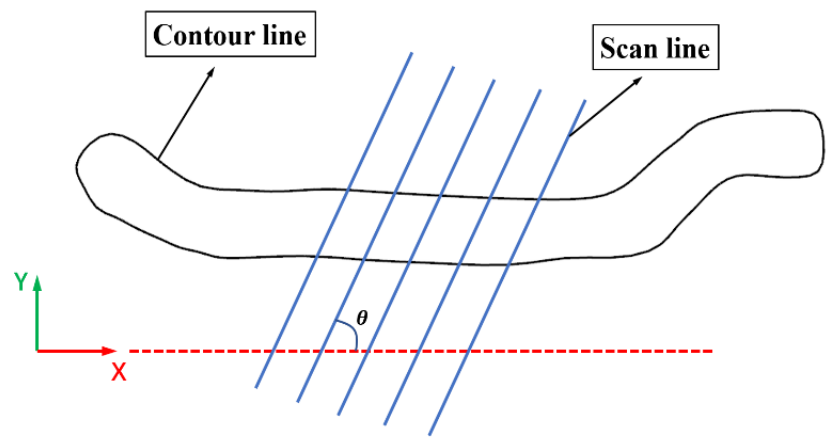

Fig. 5 The direction of the scan line 
After a series of calculations, the statistical results are shown in Fig. 6. It is easy to conclude that the interval with the minimum total length of the line segments is $[60,90)$. According to the proposed method, the relatively optimal inclination angle $\theta$ of the scan line is $75^{\circ}$. As shown in Fig. 7, for the contour lines in Fig. 5, scan lines in several directions are used to generate the internal zigzag path. It is clear from the figure that when the inclination angle is $75^{\circ}$ or $105^{\circ}$, there are almost no sharp corners in the internal zigzag path. Compared with other inclination angles, $75^{\circ}$ is a relatively ideal inclination angle, although it is not necessarily the best one. The effectiveness of the proposed method is verified by this case.

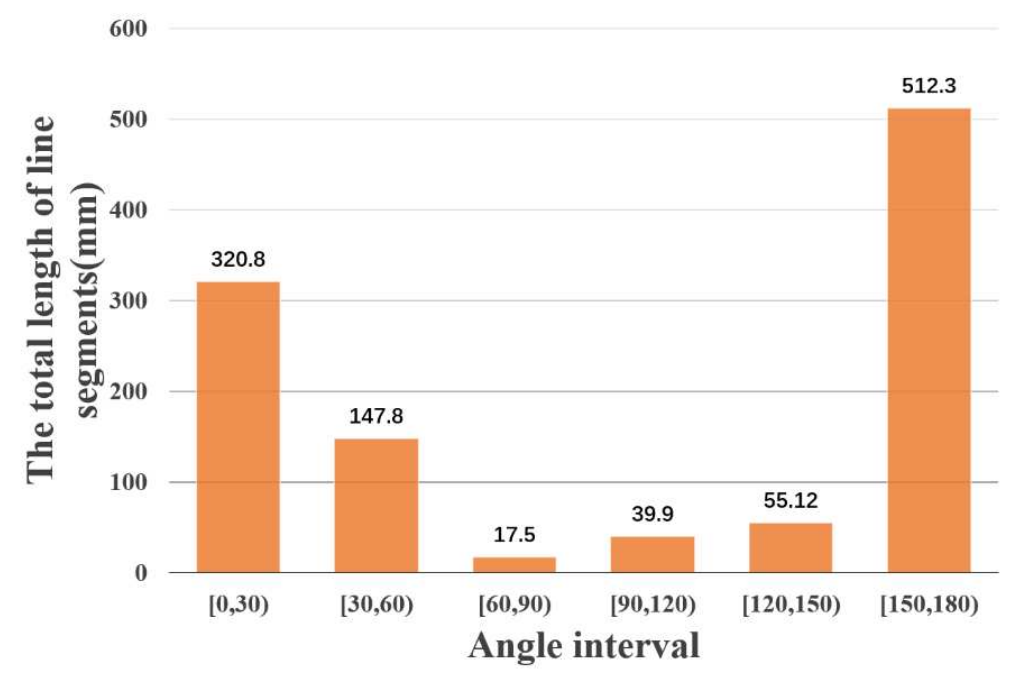

Fig. 6 The total length of line segments in each angle interval

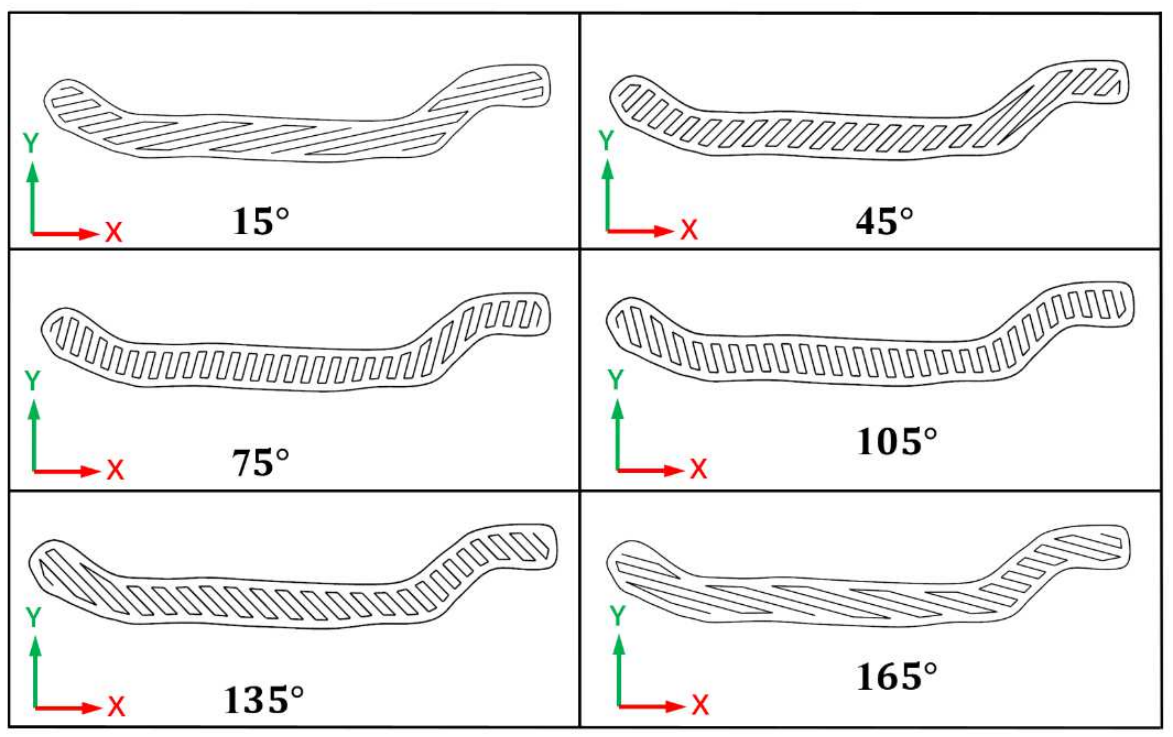

Fig. 7 Path with different inclination angles 


\section{Division and filling of the internal areas}

After the direction of the scan line is determined, the next step is to divide the internal region of the contour into several sub-regions and fill each sub-region with a continuous zigzag curve. Without loss of generality, assume that the direction of the scan line is perpendicular to the $\mathrm{x}$-axis, and the paths to fill the interior can be obtained by intersecting a number of scan lines with the offsetting contour lines. To avoid the underfilled phenomenon as illustrated in Fig. 4, the proposed tool-path generation method will accurately define the position of the sub-region. This method for division and filling of the internal areas mainly contains three steps.

Step 1: Pick out extreme points and sort them. As mentioned previously, the offsetting contour is composed of line segments. In practice, they are stored in the buffer in the form of point sets. The points of external offsetting contour lines are stored in an anti-clockwise manner, while the points of internal offsetting contour lines are stored in a clockwise manner. In the following sections, as shown in Fig. 8a, a point in the point set is called an extreme point if its $\mathrm{x}$-value is greater than or less than the $\mathrm{x}$ value of two adjacent points. Specifically, When the $x$-value of a point is equal to the $\mathrm{x}$-value of one of its adjacent points, the previous point or the next point is selected as the new adjacent point (Fig. 8b). Considering the subsequent operations, the extreme points need to be identified as concave points or convex points. Concave and convex points are defined as follows: as shown in Fig. 8c, assuming $V$ is the extreme point, $V_{1}$ is the previous point of the extreme point and $V_{2}$ is the next one. When the vector product of vector $\overrightarrow{V_{1} V}$ and $\overrightarrow{V V_{2}}$ is positive, the extreme point is a convex point, otherwise, it is a concave point. After all the extreme points of the offsetting contour are picked out, sort them according to their x-value from small to large. As shown in Fig. 9, there are 21 extreme points, including 9 convex points and 12 concave points. These extreme points divide the internal area into 8 sub-sections.

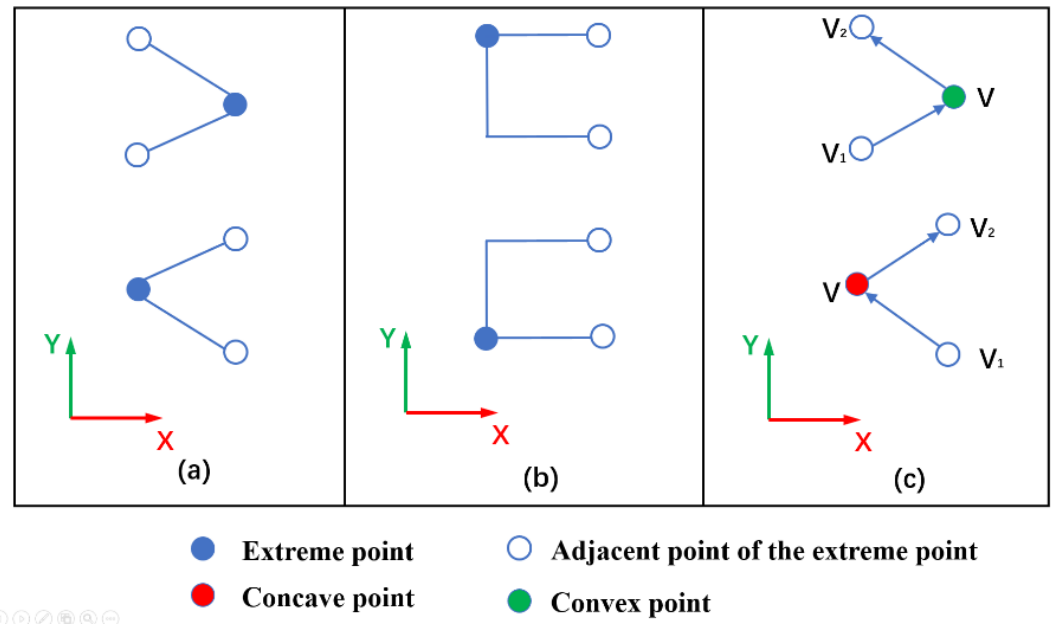

Fig. 8 Illustration of extreme point, concave point and convex point 


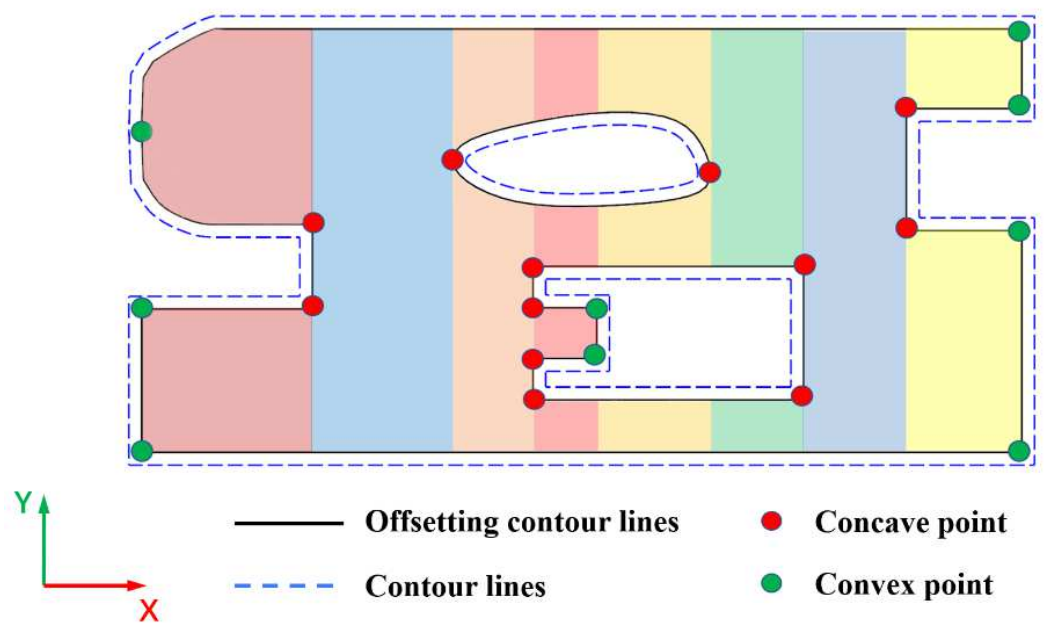

Fig. 9 Preliminary partition based on extreme points

Step 2: Determine the position of all scan lines. First of all, it can be determined that there must be a scan line at the extreme point, as shown in Fig. 10. In particular, to avoid the situation where the intersecting line segments cannot be obtained, for extreme points with the same $x$ value, if the number of convex points is odd, the scan line needs to be adjusted by a certain distance. For example, the first scan line in Fig. 10 is moved to the right by a distance of $0.1 d$ ( $d$ is the path space), because there are three convex points. Besides, the positions of other scan lines between two adjacent scan lines going through the extreme point are determined according to the path space. To ensure manufacturing quality and efficiency, process parameters in WAAR should not be changed significantly. Therefore, the path space is usually determined as a certain value before the WAAR process. Assuming that the preset path space is $d$, the distance between two adjacent scan lines going through the extreme point is $L$. Since $L$ is not always an integer multiple of $d$, a slight adjustment to $d$ is required. The specific method is as follows: When $L$ is divided by $d$, the result is $N$. If the fractional part of $N$ is less than or equal to 0.5 , then $N_{\text {new }}$ is equal to the integer part of $N$, otherwise $N_{\text {new }}$ is equal to the integer part of $N$ plus 1 . Finally, divide $L$ by $N_{\text {new }}$ to get the adjusted path space of this area.

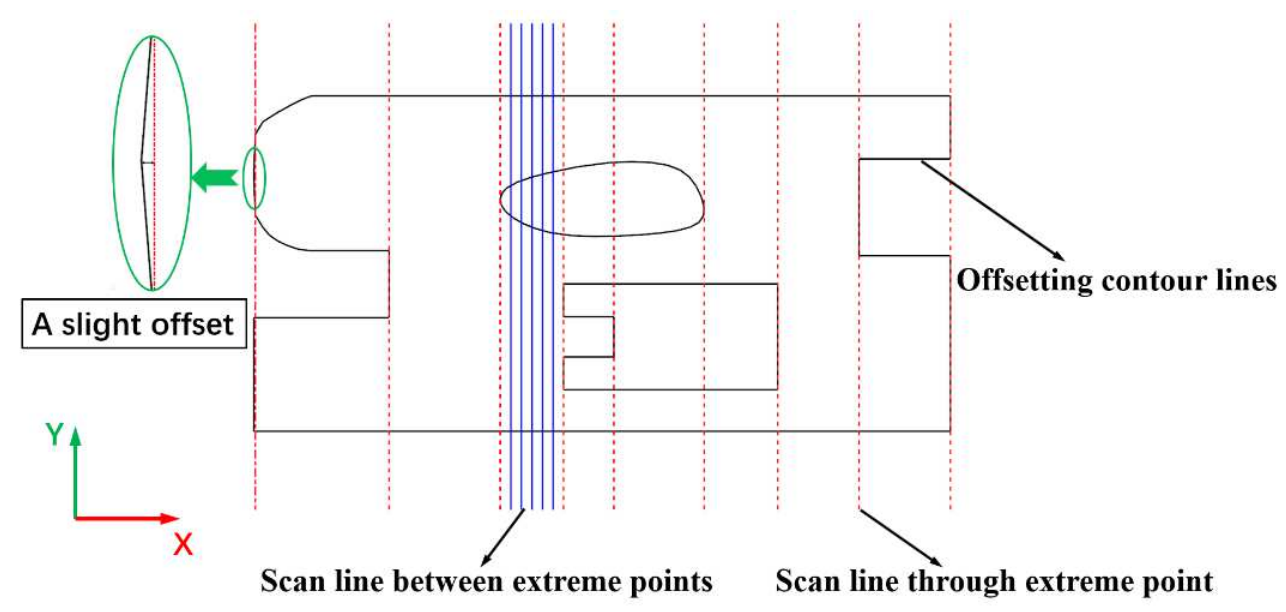

Fig. 10 Illustration of the position of the scan lines 
Step 3: Intersect the scan line with the offset contour line. The intersection point is obtained by the intersection of the scan line and each line segment of the offset contour. The intersection points are arranged in descending order of y-value, and every two intersection points form a line segment. Special treatment is required for the intersection of the offset contour line and the scan line going through the extreme point. First, if a line segment of the offset contour is parallel to the scan line, it is considered that there is no intersection point between them. The second is that if the intersection point is a concave point, it needs to be discarded. According to the number of line segments obtained after the intersection operation, each sub-section (Fig. 9) is divided into several sub-regions. In each sub-region, all the line segments can be connected as a continuous curve. As shown in Fig. 11, there are 21 sub-regions, that is, there are 21 sub-paths.

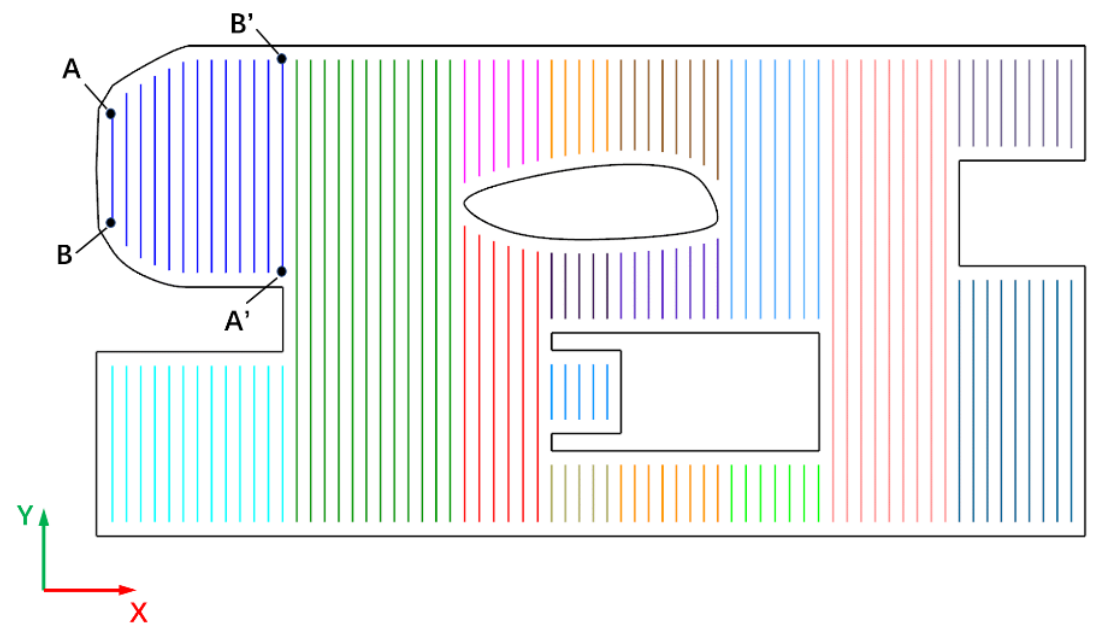

Fig. 11 Illustration of paths of each sub-region

\section{Connection of sub-paths}

In terms of deposition efficiency and quality, the less the number of sub-paths, the better. Therefore, the sub-paths should be connected as much as possible. Assume that the positive direction of the $\mathrm{x}$-axis is the filling direction of the path. As shown in Fig. 11, for each sub-path, there are two potential starting points (point $A$ and $B$ ) and two corresponding ending points (point $A^{\prime}$ and $B^{\prime}$ ). By default, the point with large $\mathrm{y}$-value (point $A$ ) is the starting point, and the corresponding ending point (point $A^{\prime}$ ) is also determined. Only the sub-paths of two adjacent sub-sections can be connected. Among the potential starting points of each sub-path of the next sub-section, the point closest to the ending point of the current sub-path is regarded as the potential connection point. Two sub-paths can be connected into a continuous curve, and the following two conditions need to be met: the first is that the connecting segment between the ending point of the current sub-path and the potential connection point of the next sub-section cannot intersect the contour lines; the second is that the length of the connection line cannot exceed a certain value. Because if the length is too long, overfilled phenomenon will occur as shown in Fig. 3. The extreme value of this length can be determined by 
the user. In the current case, twice the path space is taken as the extreme value.

In addition, there is a special case when connecting two sub-paths. As shown in Fig. 11, according to the default rules, point $A$ is the starting point and the ending point is $A^{\prime}$. However, because the second condition mentioned above cannot be met, this sub-path cannot be connected to the sub-path of the next sub-section. In view of this situation, the problem can be solved by adopting point $B$ as the starting point of the sub-path. It must be noted that in the process of forming a continuous curve, this method of changing the starting point can only be used once. As shown in Fig. 12, after the sub-paths connection process, the number of sub-paths is reduced from 21 to 6 .

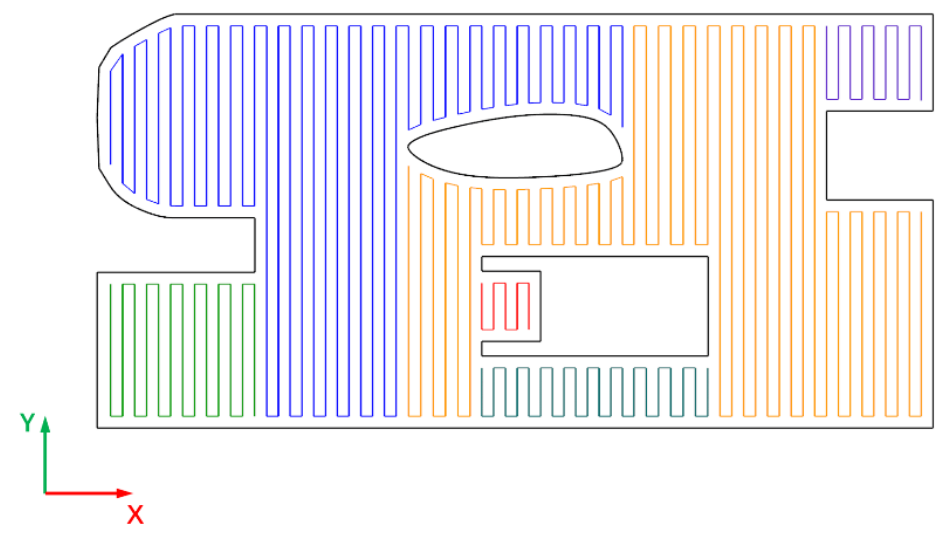

Fig. 12 Illustration of final sub-paths

\section{Implementation and discussion}

In order to demonstrate the ability of the developed algorithm to solve the underfilled problem, a simple planar deposition experiment is carried out. The experiment was implemented on a mild steel plate and the filler material is the microslag self-shielded flux cored wire with the diameter of $1.6 \mathrm{~mm}$. The shielding gas with $80 \% \mathrm{Ar}$ and $20 \% \mathrm{CO}_{2}$ was used with a flow rate of $20 \mathrm{~L} / \mathrm{min}$. The following parameters remain constant during the experiment: welding current $340 \mathrm{~A}$, welding voltage $30 \mathrm{~V}$, welding speed $8 \mathrm{~mm} / \mathrm{s}$ and path space $7 \mathrm{~mm}$. Two types of paths, generated by the general hybrid method and the proposed hybrid method, and the corresponding experimental results are presented in Fig. 13. It is clear that there are some underfilled areas in the bounded plane of deposited metal adopting the general hybrid paths. By contrast, the surface of the deposited metal adopting the proposed hybrid paths is better in terms of evenness and flatness. 


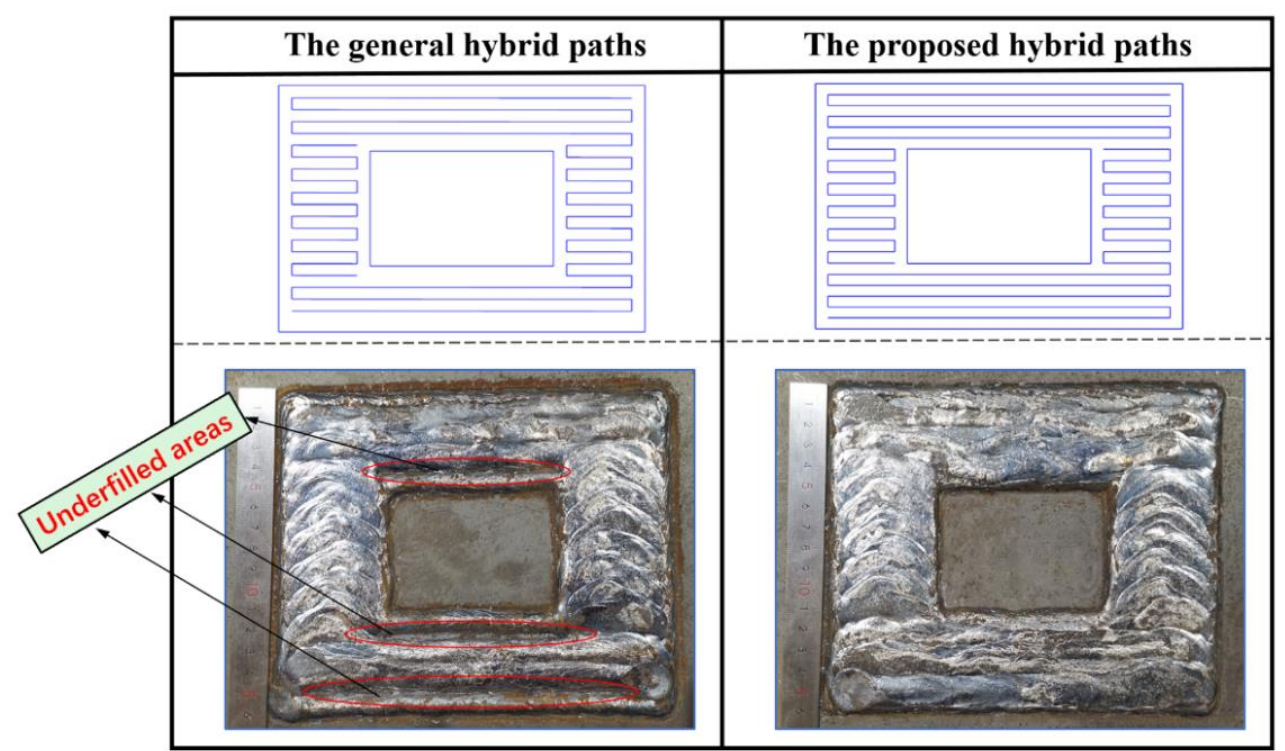

Fig. 13 Experimental comparison of the general hybrid paths and the proposed hybrid paths

In addition, to further validate the effectiveness and robustness of the developed algorithm for WAAR, four damaged hot-forging dies (blade die, turbine disk die, valve body die, crankshaft die) were repaired with WAAR technology adopting the proposed path generation method, as shown in Fig. 14. All the WAAR processes were conducted by an $\mathrm{ABB}$ robot and a Fronius MIG/MAG welding machine. During the WAAR processes, there were no incomplete fusion defects and no phenomenon of weld bead collapse. Meanwhile, all the hot-forging dies after remanufacturing show satisfied dimensional accuracy. It can be concluded that the developed algorithm is not only applicable to hot-forging dies of various geometric shapes, but also can guarantee the deposition quality and accuracy.

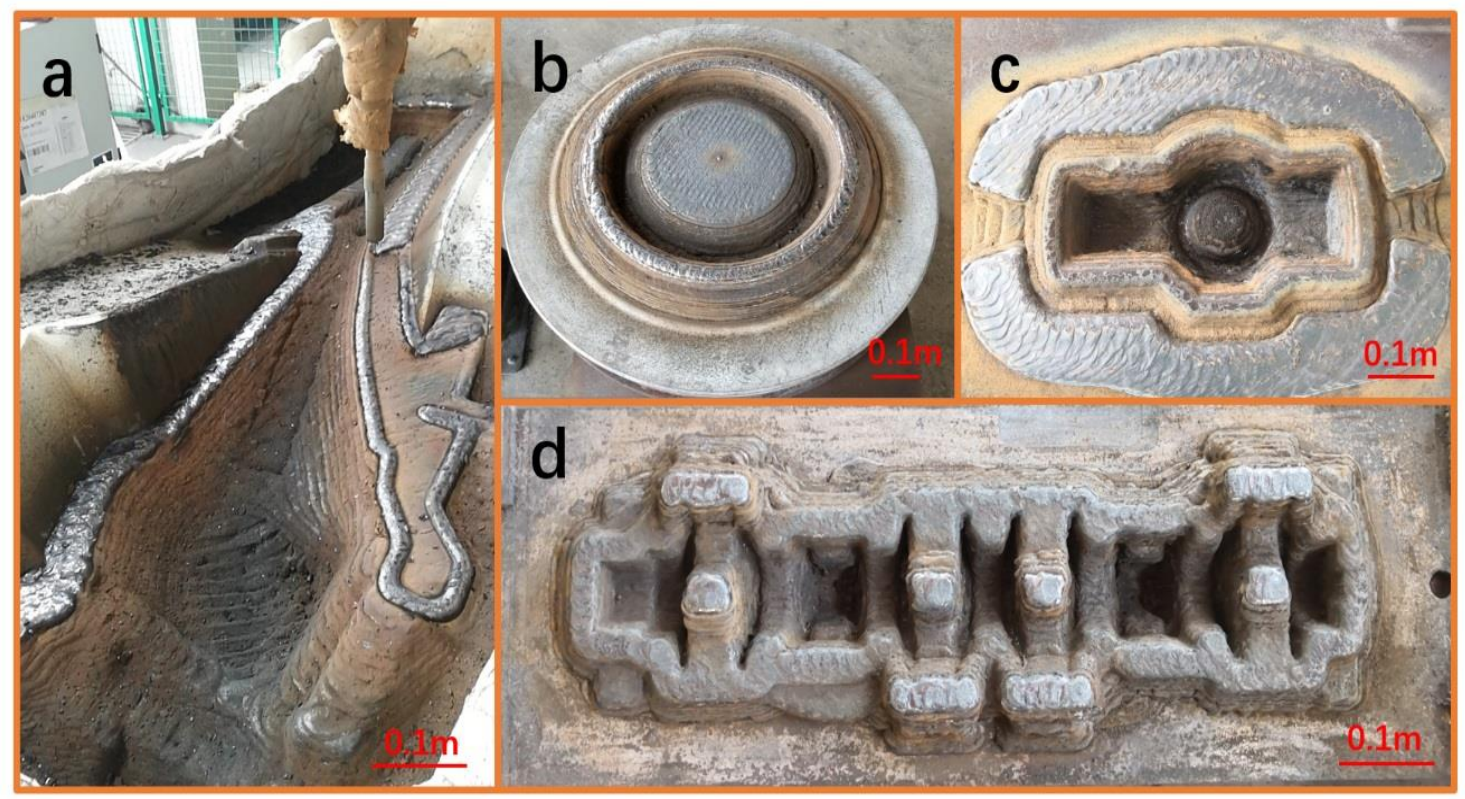

Fig. 14 Four hot-forging dies after WAAR: (a) blade die, (b) turbine disk die, (c) valve body die, (d) crankshaft die 


\section{Conclusions}

This paper presents a path generation method for the wire and arc additive remanufacturing (WAAR) process of hot-forging dies. Different from the WAAM process, the WAAR process is carried out on the gouged die with arbitrary geometric shapes and its welding heat input is generally large. With consideration of these specific characteristics, the hybrid path planning strategy is an appropriate strategy for WAAR process. The proposed hybrid path generation algorithms firstly determine the relatively optimal direction of the scan line based on the inclination angle interval with the minimum total length of the contour lines, which can greatly reduce the appearance of sharp angles. And then, the internal region is divided into several sub-regions according to the location of the extreme points and make an adaptive adjustment to the path space for each sub-region. These operations can prevent the occurrence of underfilled phenomenon. At last, the number of sub-paths is greatly decreased by adopting the proposed strategy for interconnection. Through a comparative experiment, the proposed hybrid paths can obtain a flatter surface of the deposited metal than the general hybrid paths. Furthermore, the repair trials of four damaged hot-forging dies with WAAR technology validate the effectiveness and robustness of the developed algorithm.

\section{Acknowledgements}

The authors gratefully acknowledge a Project Funded by the Priority Academic Program Development of Jiangsu Higher Education Institutions (PAPD).

\section{Declarations}

Author contribution Yonghua Shen: conceptualization, experiments, writing; Yanhong Wei: conceptualization, writing and editing; Renpei Liu: conceptualization and project supervision.

Funding Not applicable.

Competing interests The authors declare no competing interests.

Availability of data and material Not applicable.

Ethics approval Not applicable.

Consent to participate Not applicable.

Consent for publication Not applicable. 


\section{References}

[1] Debroy, Tarasankar, et al. "Additive manufacturing of metallic components - Process, structure and properties." Progress in Materials Science $92(2018)$ : pp. $112-224$.

[2] Lewandowski, John J. , and M. Seifi . "Metal Additive Manufacturing: A Review of Mechanical Properties." Annual Review of Materials Research 46(2016) :151186.

[3] Tapia, Gustavo, and A. Elwany . "A Review on Process Monitoring and Control in Metal-Based Additive Manufacturing." Journal of Manufacturing Science \& Engineering 136.6(2014):060801.

[4] Herzog, Dirk, et al. "Additive manufacturing of metals." Acta Materialia 117 (2017).

[5] Karaye1, Elif, and Y. Bozkurt. "Additive manufacturing method and different welding applications." Journal of Materials Research and Technology 9. $5(2020): 11424-11438$.

[6] Jörn Mehnen, et al. "Design study for wire and arc additive manufacture." International Journal of Product Development 19.1/2/3(2014):2-20.

[7] Wang, Fude , S. Williams, and Paul Colegrove.... "Microstructure and Mechanical Properties of Wire and Arc Additive Manufactured Ti-6Al-4V." Metallurgical \& Materials Transactions Part A 44.2(2013):968-977.

[8] Xiong, Jun , et al. "Fabrication of inclined thin-walled parts in multilayer single-pass GMAW-based additive manufacturing with flat position deposition." Journal of Materials Processing Technology 240 (2017):397-403.

[9] S., et al. "Wire + Arc Additive Manufacturing." Materials Science and Technology 32.7(2016):641-647.

[10]Li Y, Han Q , Zhang G, et al. A layers-overlapping strategy for robotic wire and arc additive manufacturing of multi-layer multi-bead components with homogeneous layers[J]. International Journal of Advanced Manufacturing Technology, 2018.

[11]Ding D , Shen C, Pan Z, et al. Towards an automated robotic arc-weldingbased additive manufacturing system from CAD to finished part[J]. ComputerAided Design, 2016, 73:66-75.

[12]Ding, Donghong, et al. "A practical path planning methodology for wire and arc additive manufacturing of thin-walled structures." Robotics \& Computer Integrated Manufacturing 34. aug. (2015):8-19.

[13]Zhang, Yu Ming, et al. "Weld deposition-based rapid prototyping: a preliminary study." Journal of Materials Processing Technology 135.2$3(2003): 347-357$.

[14]Ding, Donghong, et al. "A tool-path generation strategy for wire and arc additive manufacturing." International Journal of Advanced Manufacturing Technology 73. 1-4 (2014) :173-183. 
[15]Dwivedi, Rajeev, and R. Kovacevic . "Automated torch path planning using polygon subdivision for solid freeform fabrication based on welding." Journal of Manufacturing Systems 23.4(2004):278-291.

[16]Venturini, Giuseppe , et al. "Optimization of WAAM Deposition Patterns for T-crossing Features." Procedia Cirp 55. Complete(2016) :95-100.

[17]Miche1, Florent, et al. "A modular path planning solution for Wire + Arc Additive Manufacturing." Robotics and Computer-Integrated Manufacturing 60. DEC. (2019) :1-11.

[18]Chen, Changrong , et al. "A review on remanufacture of dies and moulds." Journal of Cleaner Production 64(2014) :13-23.

[19] Jhavar, S. , C. P. Paul , and N. K. Jain . "Causes of failure and repairing options for dies and molds: A review." Engineering Failure Analysis 34. 8 (2013) :519-535.

[20]Liu, Dashuang , et al. "Influence of Carbon Content on Shock Hardening Behavior of Cobalt-Base Hardfacing Deposits." Materials and Manufacturing Processes 28. 6 (2013) :643-649.

[21]Jin, Yu An, et al. "A parallel-based path generation method for fused deposition modeling." The International Journal of Advanced Manufacturing Technology (2015).

[22]Jin, Yu An , et al. "Optimization of tool-path generation for material extrusion-based additive manufacturing technology." Additive Manufacturing $1-4(2014): 32-47$. 
Figures

\begin{tabular}{|l|l|}
\hline (a) (b) & (b) \\
\hline (c) & (d) \\
\hline 3D scanning & (f) \\
\hline WAAR model & \\
\hline Simulation & (h) \\
\hline
\end{tabular}

Figure 1

The WAAR process of the hot-forging die 


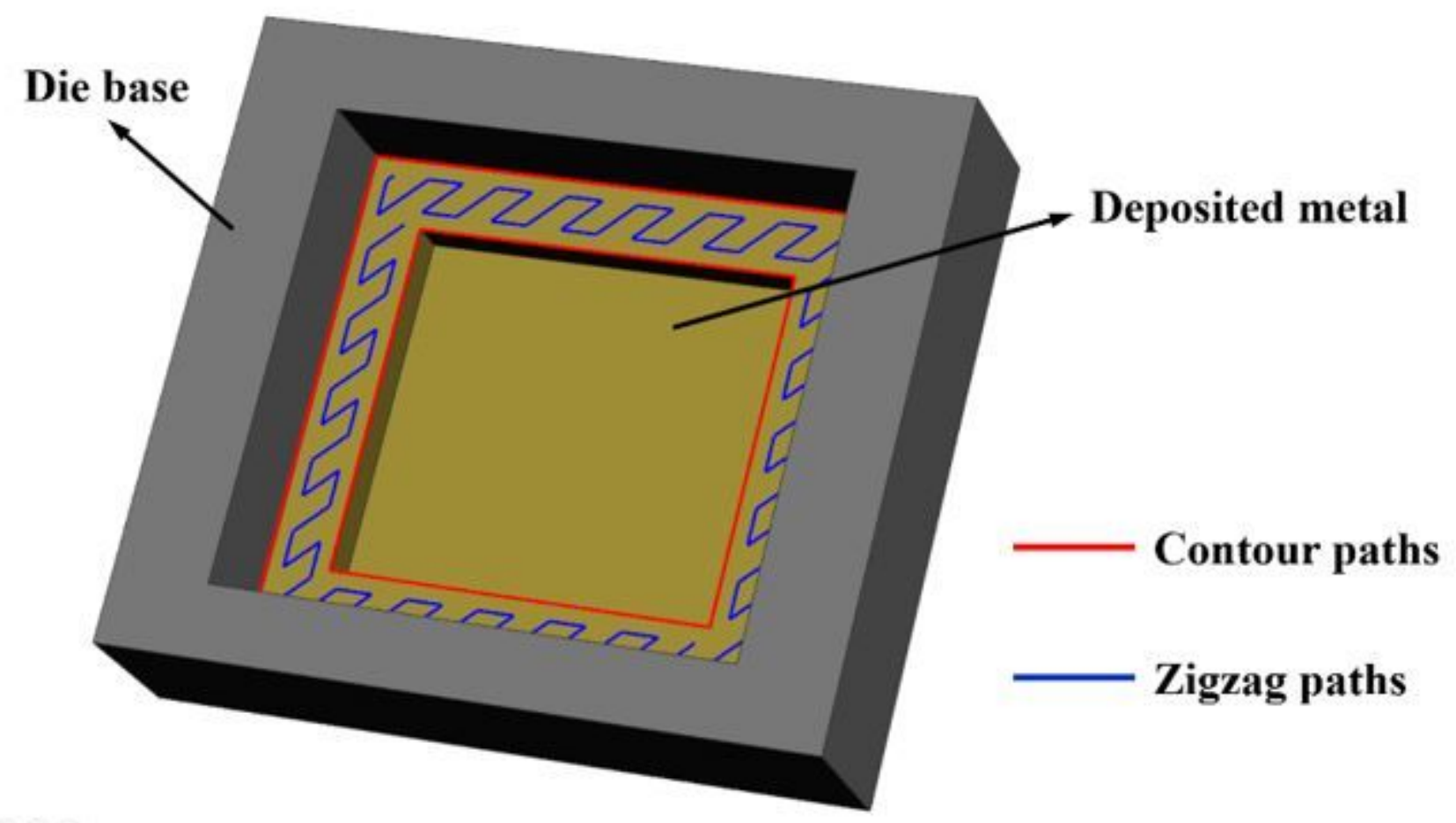

Figure 2

The hybrid path planning strategy

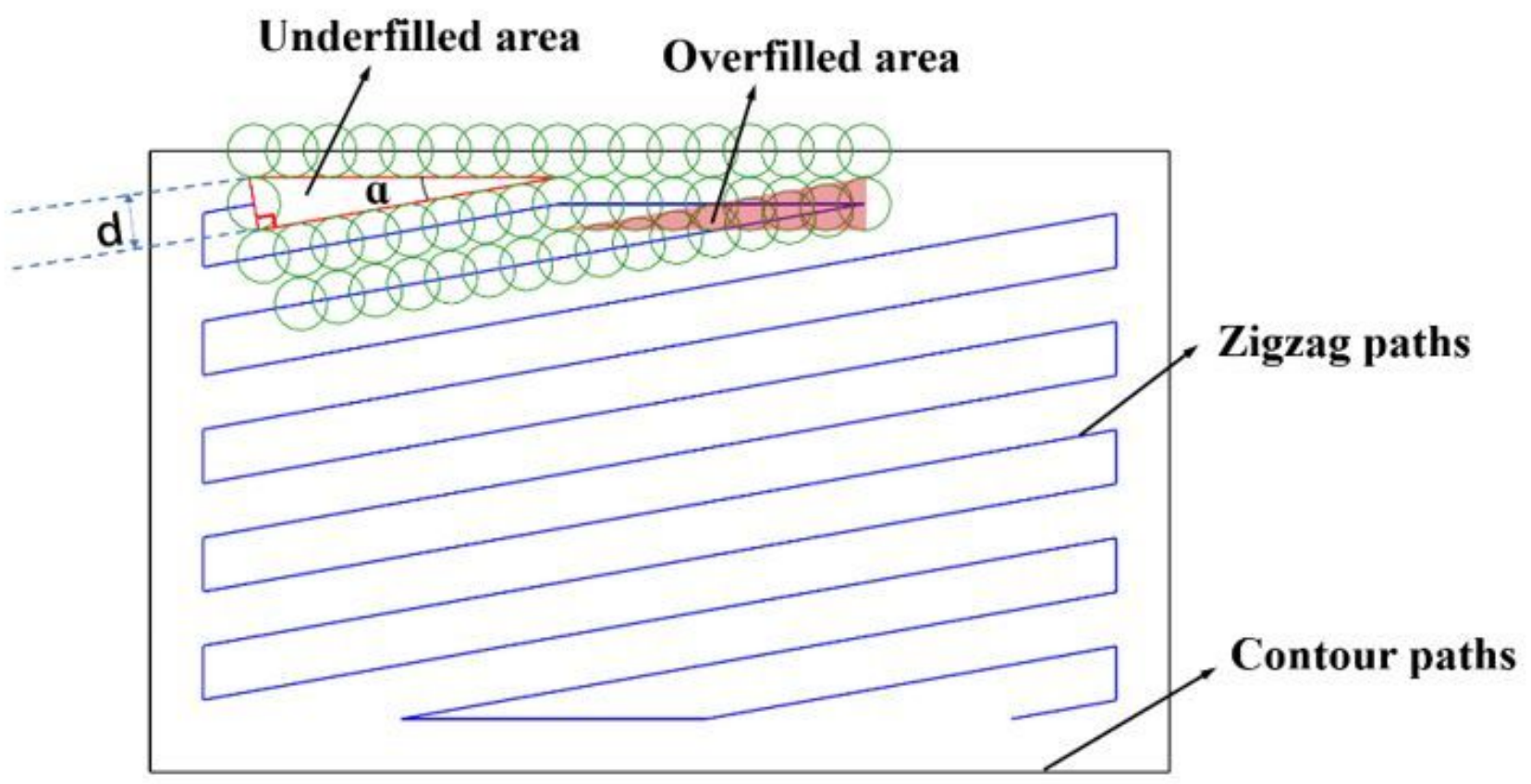

Figure 3

The overfilled and underfilled phenomenon in corners 


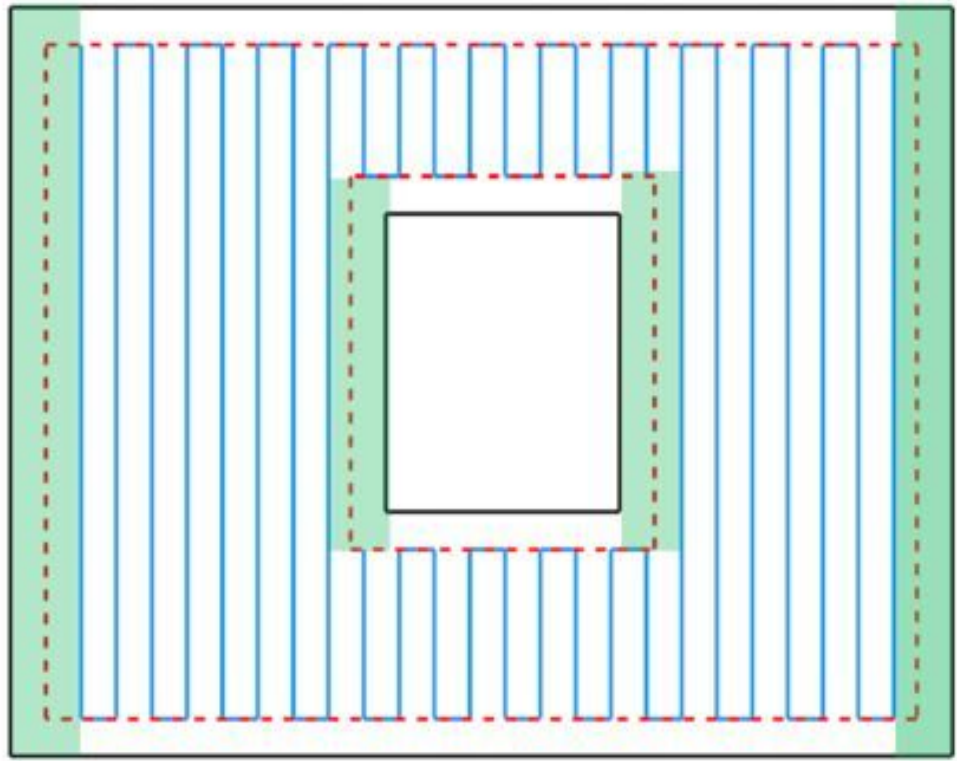

— Contour line(path)

..... Offsetting contour line

Internal zigzag path

Underfilled area

Figure 4

Illustration of the underfilled phenomenon

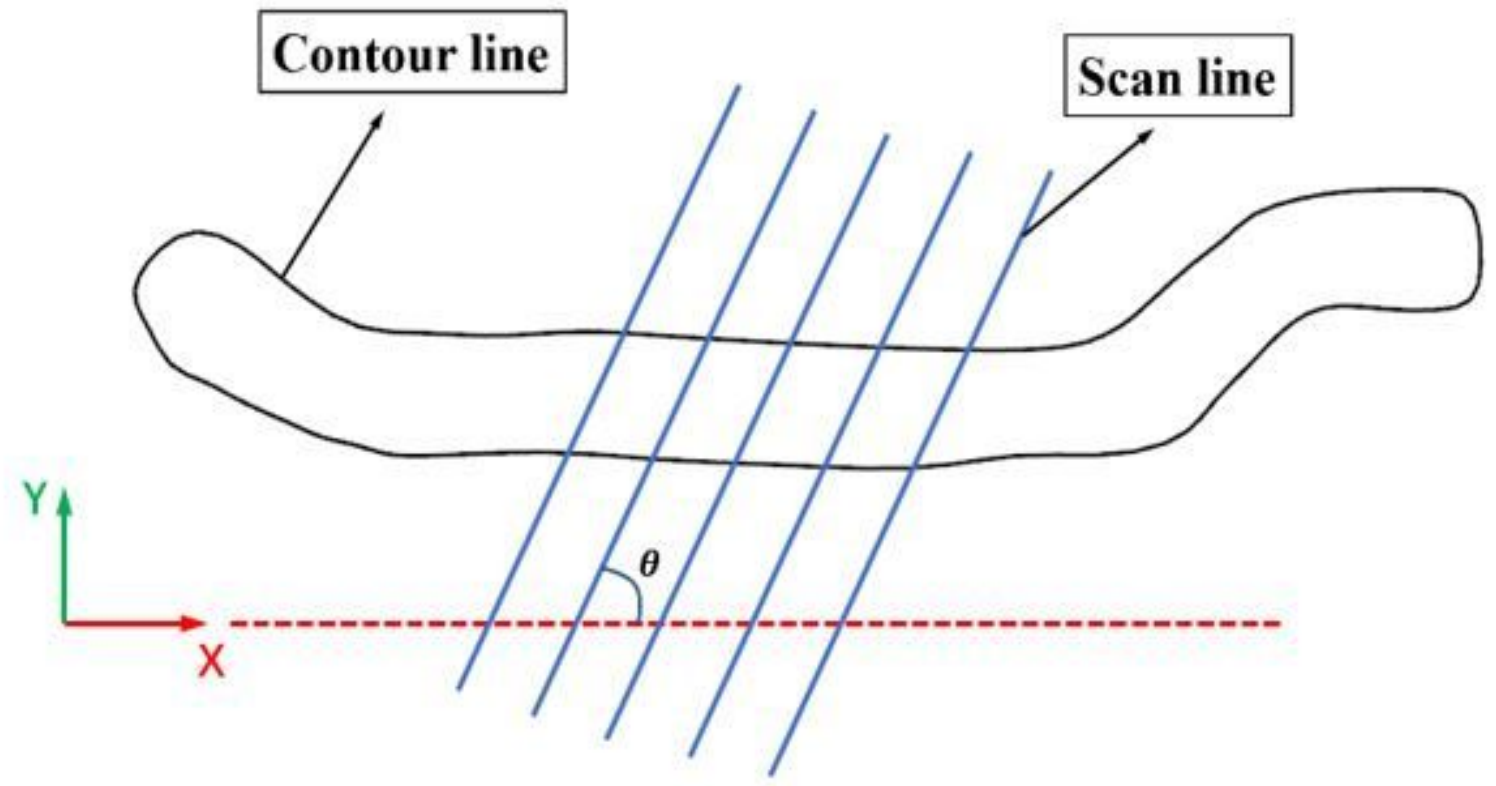

Figure 5

The direction of the scan line 
600

512.3

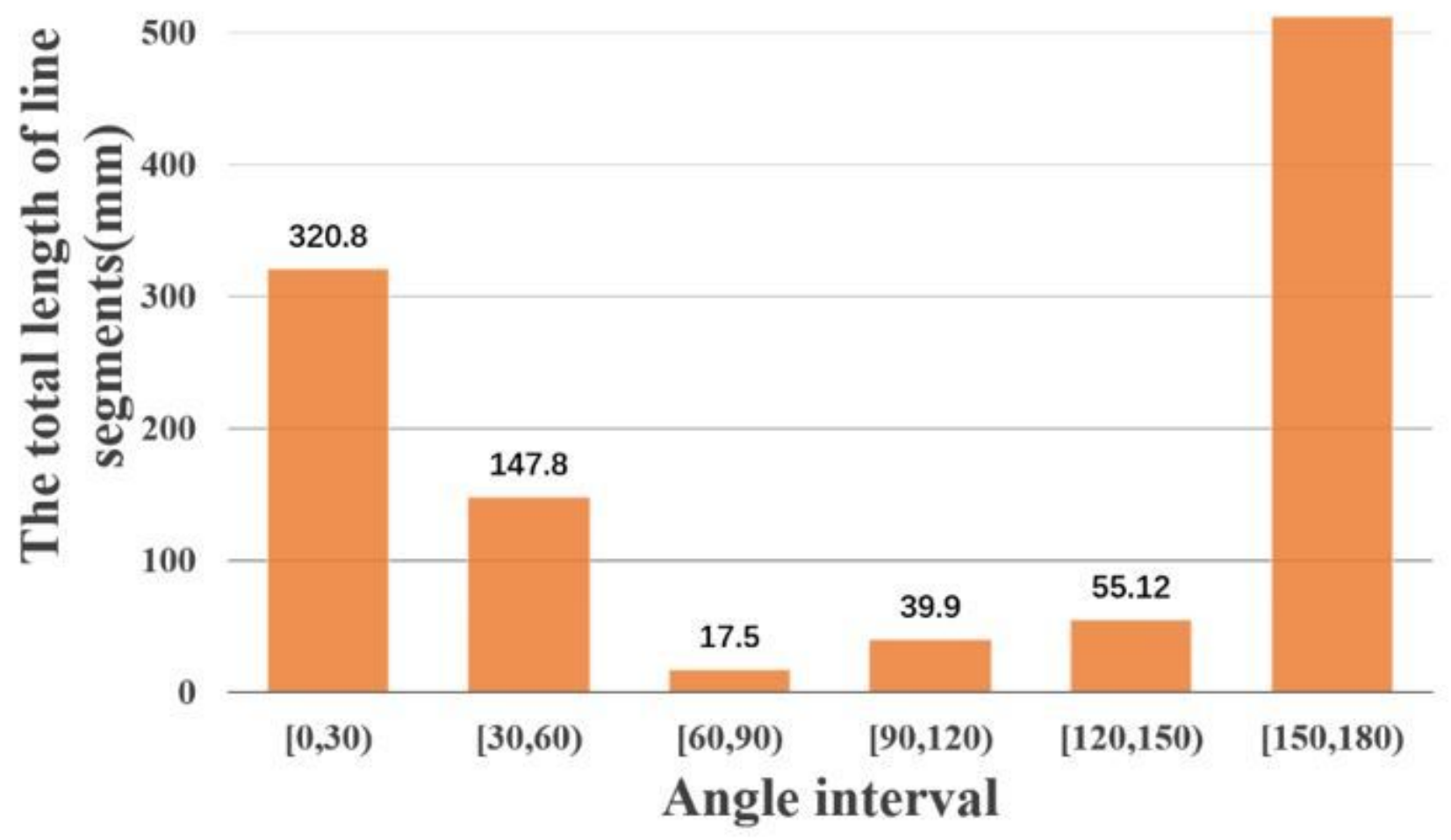

Figure 6

The total length of line segments in each angle interval 


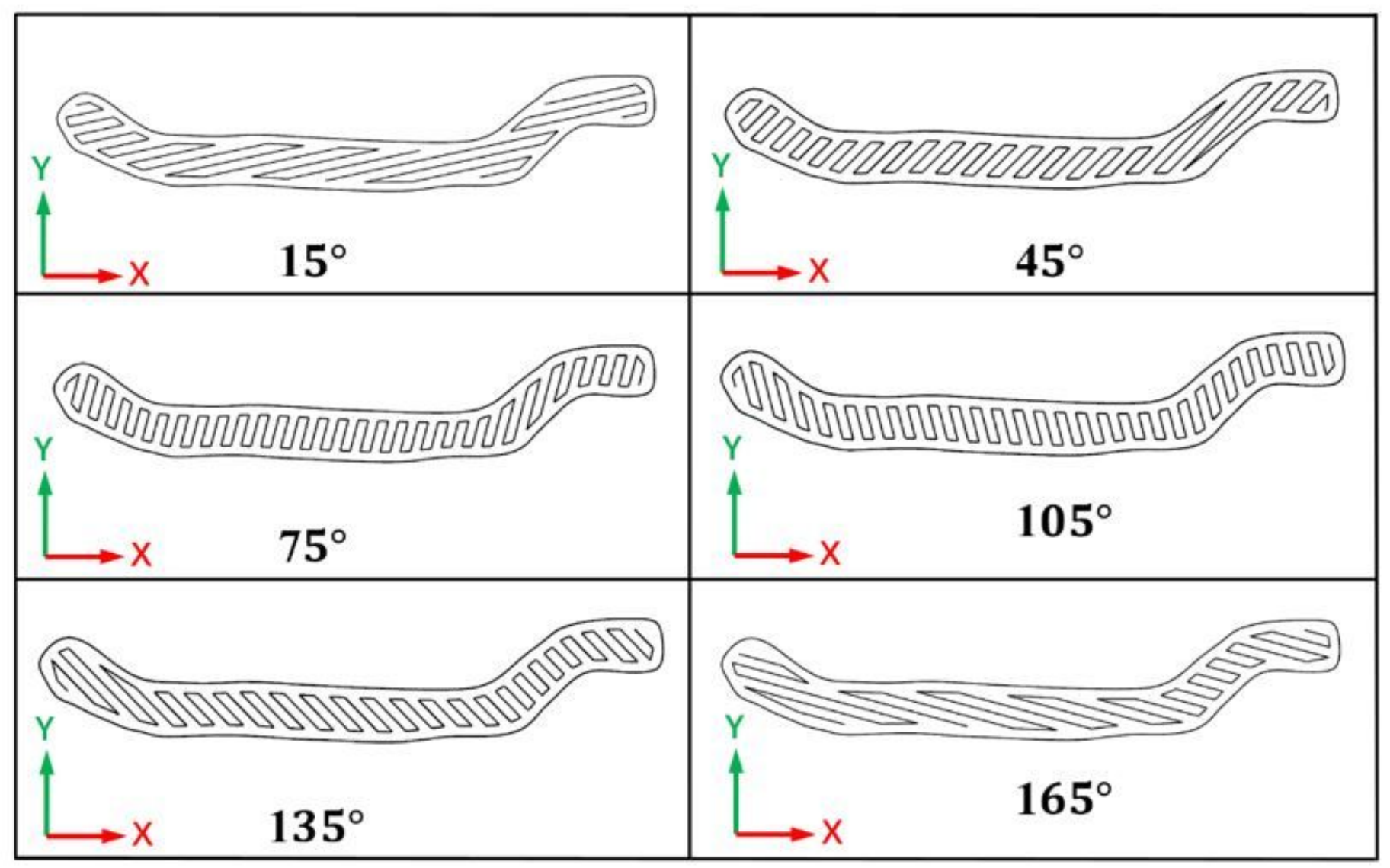

Figure 7

Path with different inclination angles

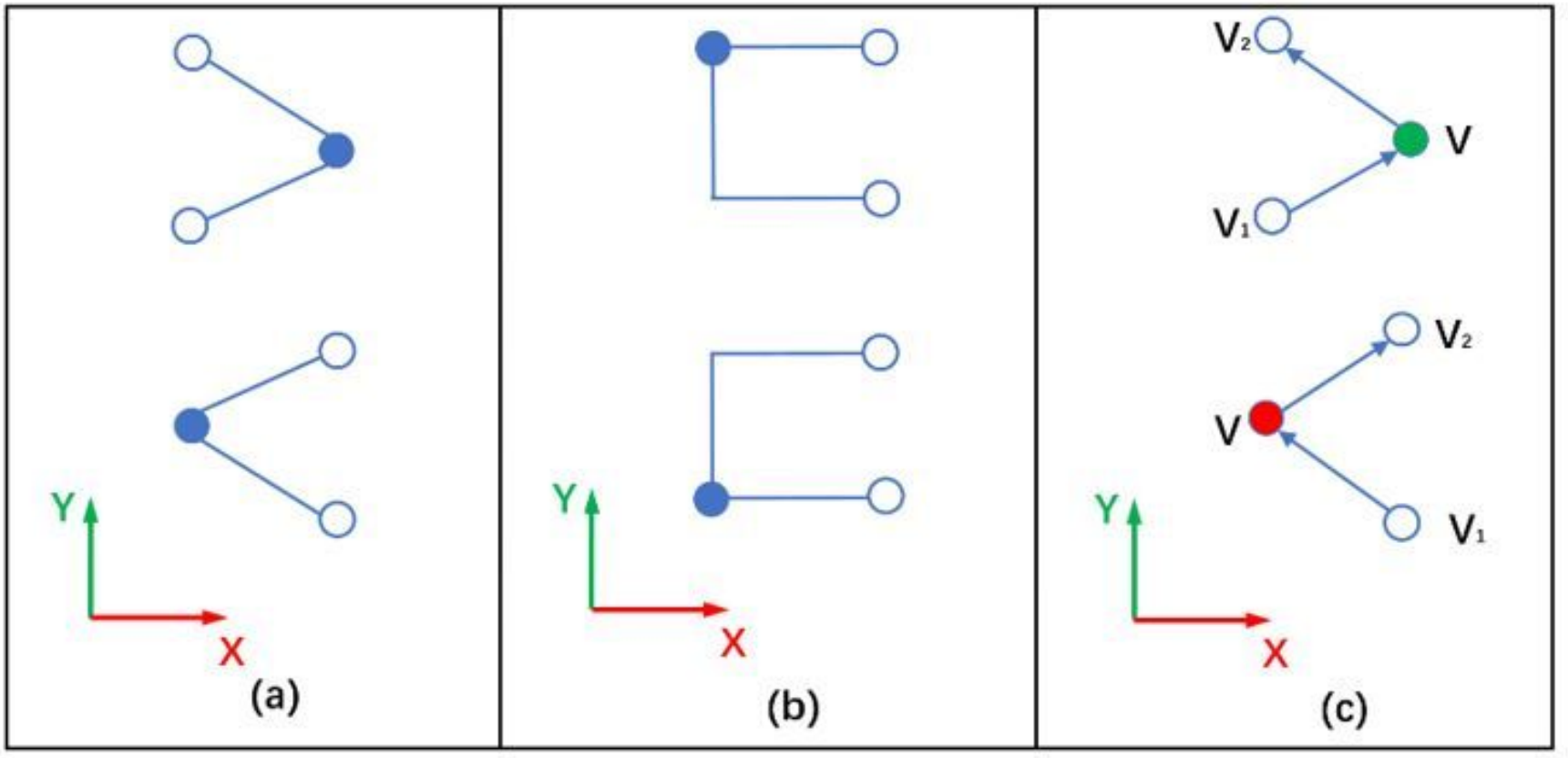

Extreme point

- Concave point $\bigcirc$ Adjacent point of the extreme point Convex point 
Figure 8

Illustration of extreme point, concave point and convex point
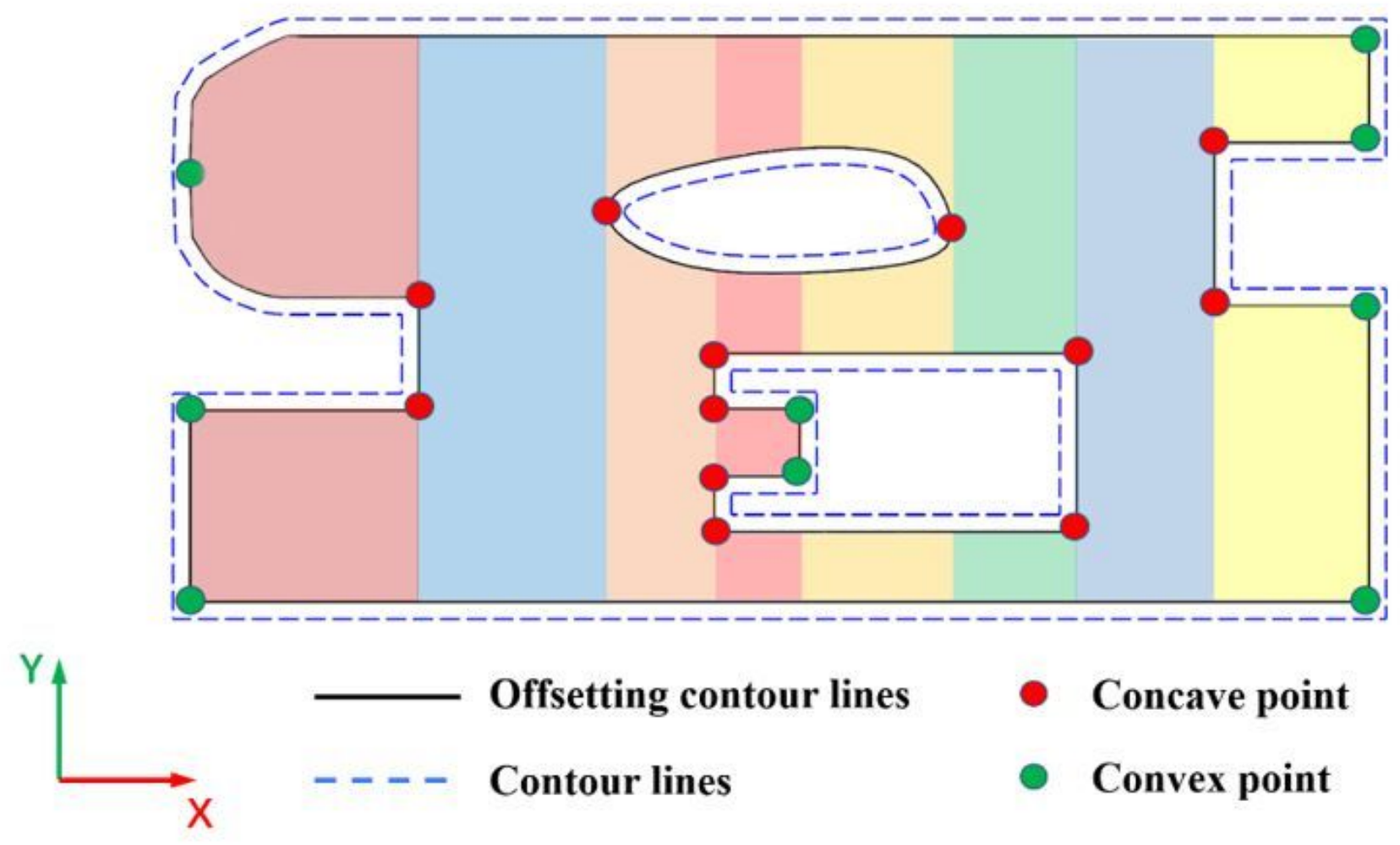

Figure 9

Preliminary partition based on extreme points

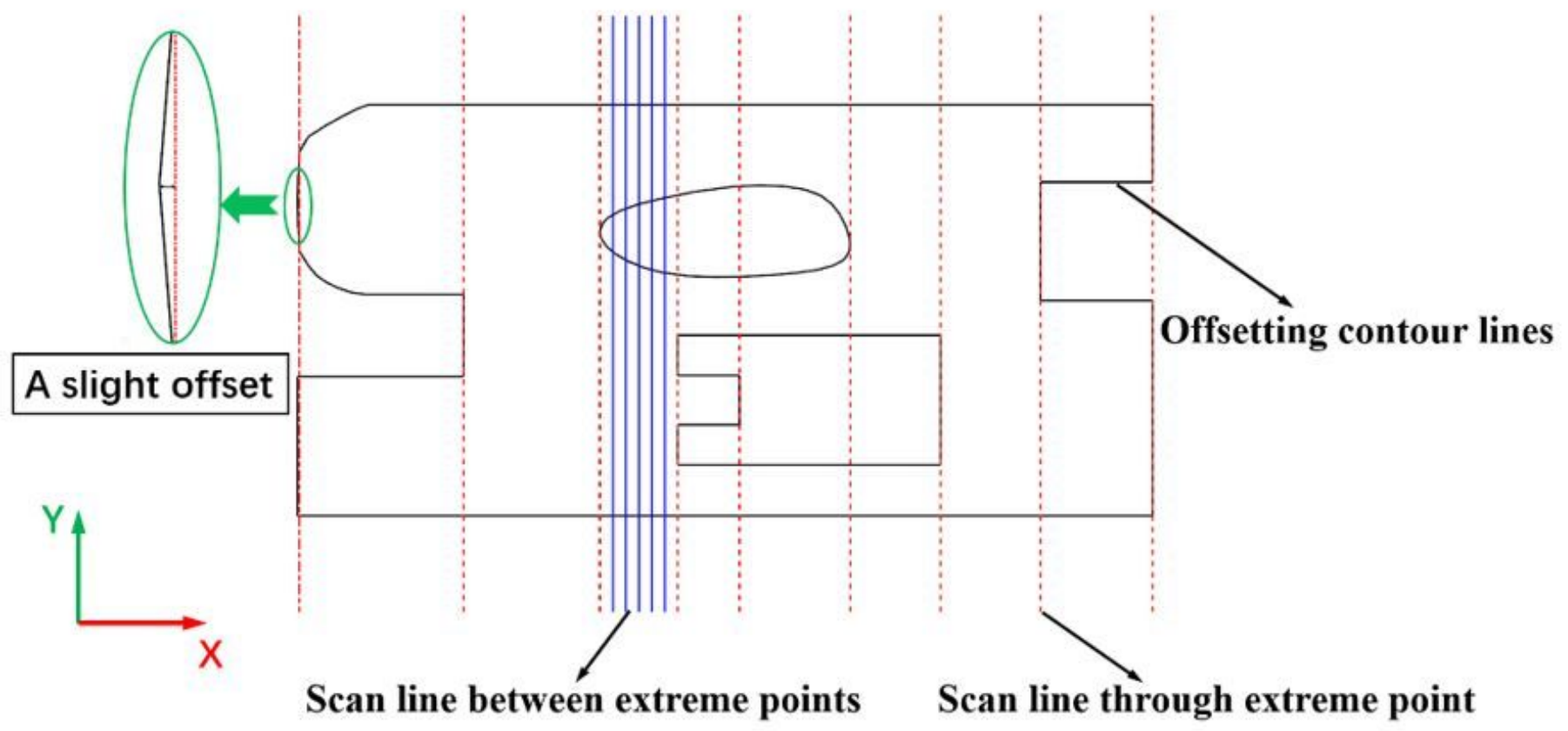

Figure 10 
Illustration of the position of the scan lines

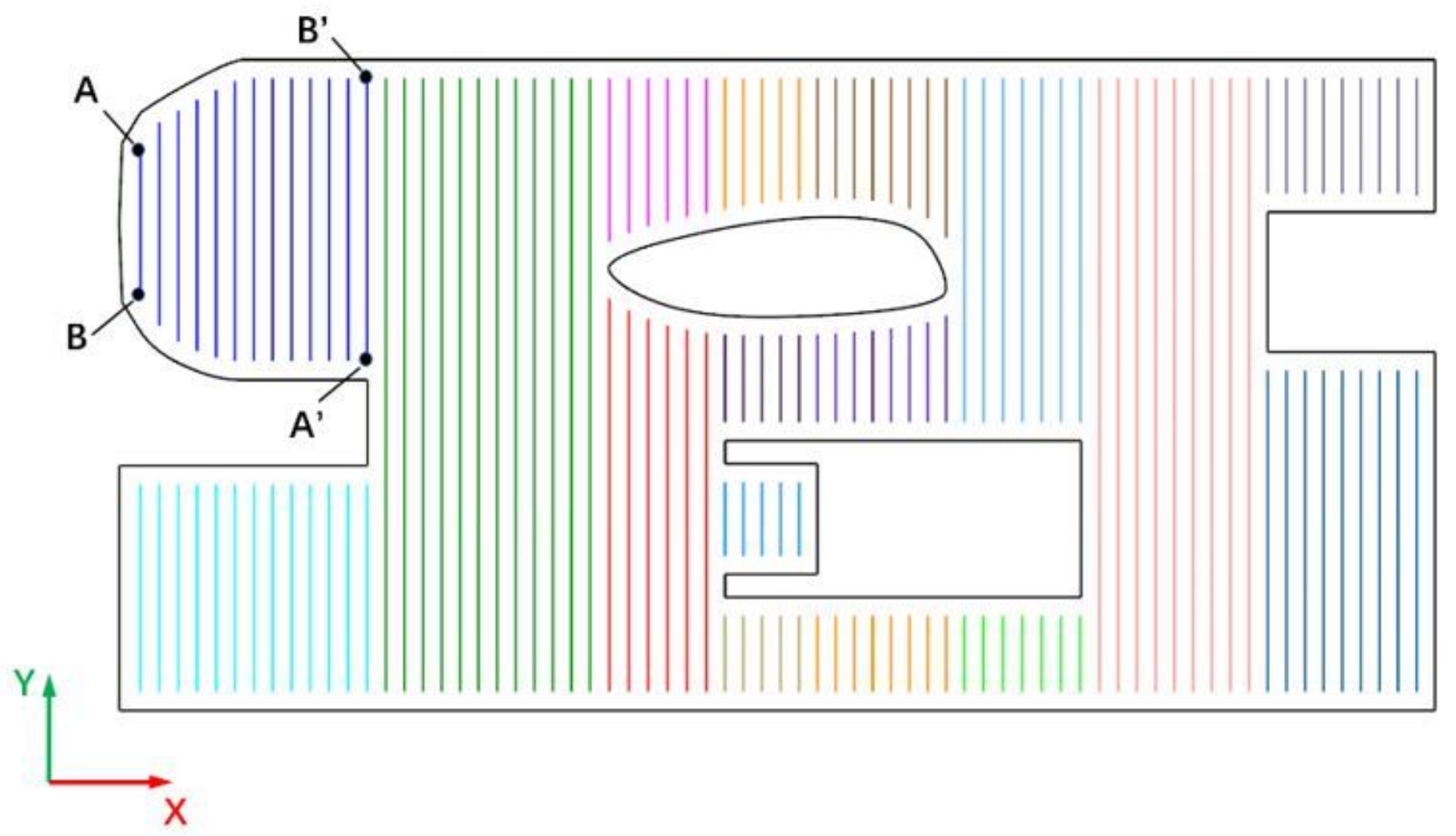

Figure 11

Illustration of paths of each sub-region

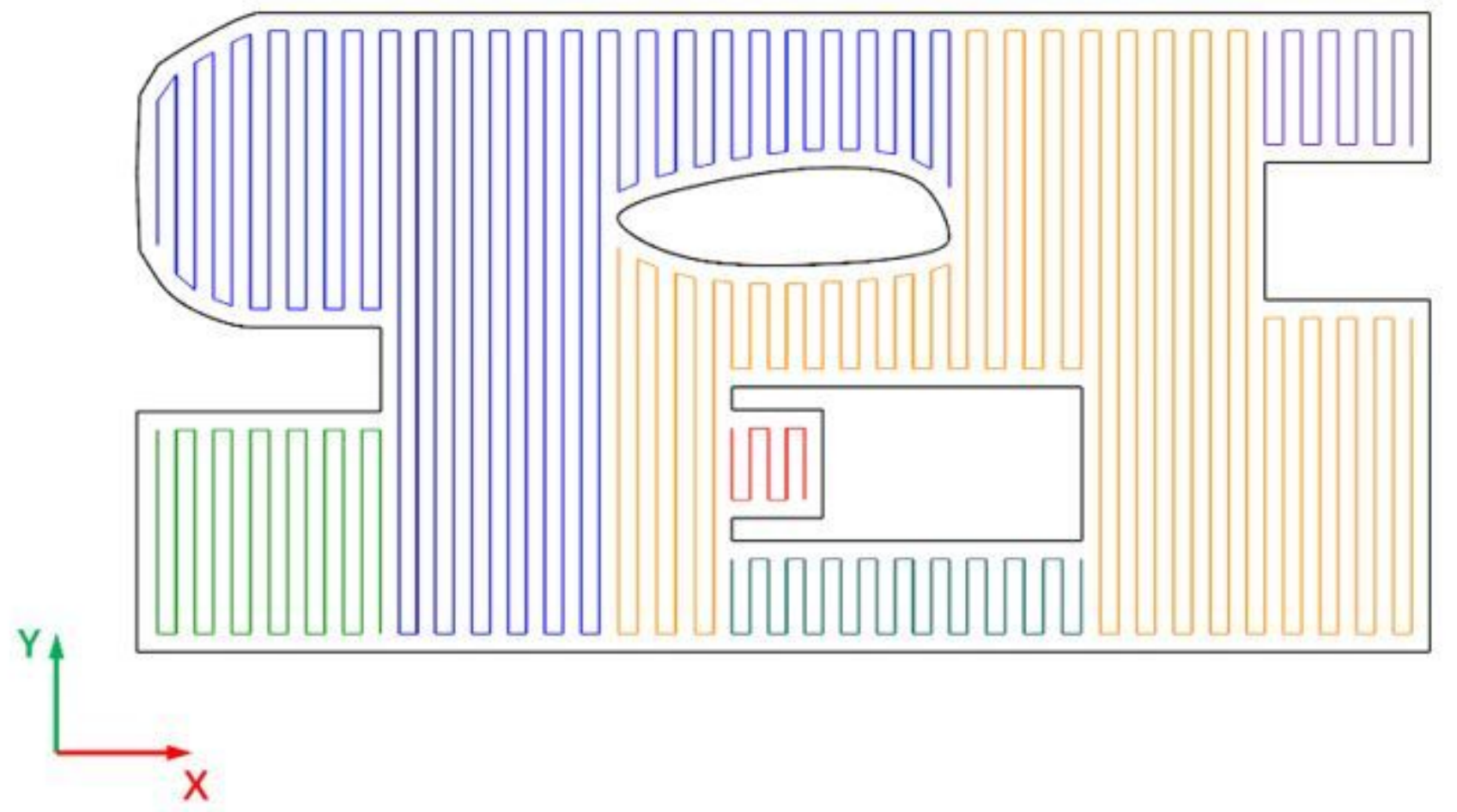

Figure 12 


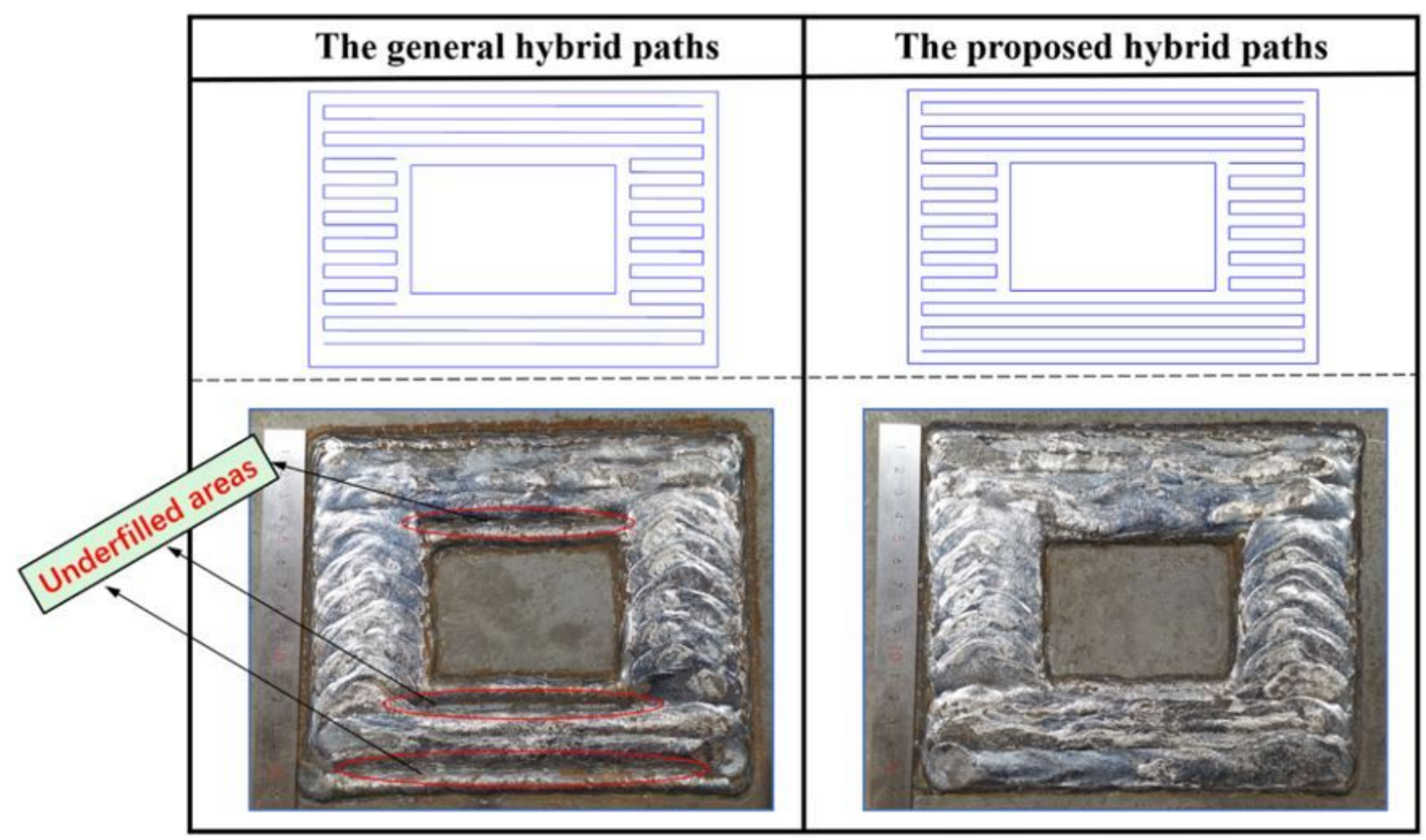

Figure 13

Experimental comparison of the general hybrid paths and the proposed hybrid paths 


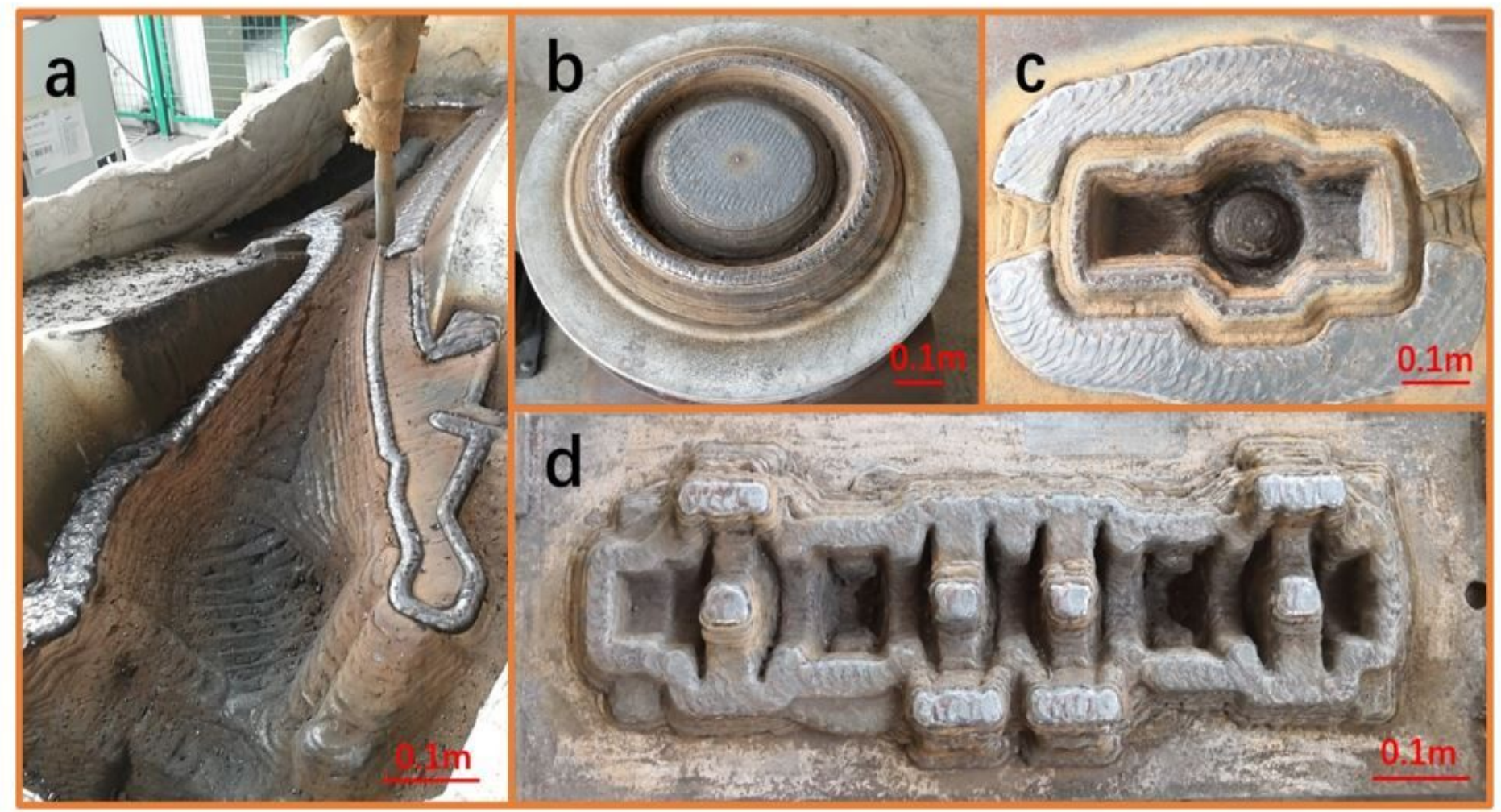

Figure 14

Four hot-forging dies after WAAR: (a) blade die, (b) turbine disk die, (c) valve body die, (d) crankshaft die 